\title{
Spatial and Temporal Properties of Gaze Return Fixations While Viewing Affective Images
}

\author{
Lubov Podladchikova, Dmitry Shaposhnikov", Tatiana Koltunova, Dmitry Lazurenko, \\ Valery Kiroy
}

Centre of Neurotechnology, Southern Federal University, Rostov-on-Don, Russia

Email address:

dgshaposhnikov@sfedu.ru (D. Shaposhnikov)

${ }^{*}$ Corresponding author

\section{To cite this article:}

Lubov Podladchikova, Dmitry Shaposhnikov, Tatiana Koltunova, Dmitry Lazurenko, Valery Kiroy. Spatial and Temporal Properties of Gaze Return Fixations While Viewing Affective Images. Advances in Applied Physiology. Vol. 5, No. 2, 2020, pp. 42-47.

doi: 10.11648/j.aap.20200502.16

Received: October 23, 2020; Accepted: November 7, 2020; Published: November 24, 2020

\begin{abstract}
Spatial and temporal properties of gaze return fixations on recently viewed regions during free viewing of affective images from The International Affective System (IAPS) image database are considered. In each test, 10 positive, 10 negative, and 10 neutral images were presented for each Subject $(n=20)$. It was revealed that the probability of the return fixations varied from 0,04 up to 0,42 for different Subjects and images (the average probability for negative, neutral and positive images was equal to $0,090 \pm 0,009,0,079 \pm 0,012$ and $0,076 \pm 0,009$ ). It was shown that the distributions of return fixations duration and preceding (returning) saccades amplitude had a bimodal character. Besides, a tendency to increase the return fixation duration with a decrease in amplitude of the preceding saccades was revealed. Return fixations which located inside the areas of interest determined by analysis of the spatial distribution of all fixations (both regular and return ones) had maximal density and duration as compared with the other image regions. Finally, the volunteers with dominant focal viewing scan-paths had a higher probability of return fixations than those with scanning trajectories $(0,09 \pm 0,003$ and $0,05 \pm 0,006)$. On the base of the obtained results, the opportunity to estimate the dominant component of visual attention during the current stage of the dynamic process of image viewing and prospective studies of return fixations have been discussed.
\end{abstract}

Keywords: IAPS Image Databases, Eye Movements, Return Gaze Fixations, Areas of Interest, Duration and Density of Fixations, Saccade Amplitude, Focal and Scanning Viewing Scan-Paths

\section{Introduction}

In many studies, the structure of the viewing scan-paths of images and scenes is considered as an important key to understanding the mechanisms of visual attention [1-6]. Despite intensive research of this problem using experimental methods and mathematical modelling, many of its fundamental aspects remain unresolved. One of these tasks is searching for the parameters of eye movements, which make it possible to determine the contribution of the dominant component of visual attention and assess the visual task to be solved at the current stage of the dynamic process of image viewing $[7,8]$.

One approach to solving this problem is to study the local elements of the viewing trajectories, in particular, the return gaze fixations on the recently viewed image areas. More and more facts are accumulating about the phenomena of Facilitation of Return [5, 9-15], but up to now the concept of Inhibition of Return has dominated [7, 16]. The oculomotor, endogenous, and exogenous factors that determine the occurrence of the phenomenon of inhibition of return, its spatial and temporal properties are analyzed in detail [7, 1012, 17-20]. Much less attention is paid to the study of facilitation of return phenomenon.

It is shown that the ratio of the phenomena of facilitation and inhibition of return depends on many factors, including the visual task, the nature of the stimuli used in the experiment, instruction before the test, the method of recording the response of the subjects. The ratio is regulated by the mechanisms of the bottom-up and top-down levels [13-15, 17, $18,21]$. It is assumed that return-inhibiting mechanisms 
dominate to solve visual search problems and examine simple images [7, 11, 20, 22]. Instead, the phenomenon of facilitating return often occurs in the visualization of complex images and the resolution of complex visual tasks [9, 12].

It is known that one of the leading factors in the regulation of visual attention in solving various visual tasks is the nature of the emotional impact of the image on a person. A study of various aspects of the perception of emotions indicates the priority of emotionally significant images compared with emotionally neutral stimuli [21, 23-29]. No studies are known to investigate the facilitation of return on presentation of emotionally significant images.

The present work is aimed at searching for return gaze fixations on recently viewed areas during free viewing of images from the International Affective Picture System, IAPS [30] with different emotional valence (positive, negative and neutral) and analysis of their spatial and temporal properties. In similar works, when images of a different type are used, without assessing their emotional impact, the properties of return saccades and the duration of gaze fixations that occur before return saccades were analyzed [10-12]. Taking into account, the results of our previous study on the features of the duration of fixations in their sequence in the vicinity of returning saccades [5] during the free viewing of reproductions of paintings used in the work of A. Yarbus [6], in our work, the fixations arising after returning saccades.

\section{Methods}

In the tests involved 20 volunteers ( 14 women, 6 men) aged 18 to 38 years (mean age 21,6 65,2 years). All subjects had normal visual acuity (or corrected to normal) and did not have a diagnosis of neurological or mental illness. Each subject signed consent to participate in tests that were carried out in compliance with the rules of bioethics, the protocol of the experiments was approved by the bioethics commission of the Southern Federal University.

The choice of the IAPS image database in our study was determined by the fact that the images in it were classified using the Self-Assessment Manikin (SAM) method according to quantitative criteria in the scales of evoked emotions [30, 31]. Initially [30, 31], on a large sample of subjects, the images were ranked on a nine-point scale for each of the three parameters, namely: Valence, Arousal, and Dominance. The criterion for classifying images according to these parameters was the subject's assessment of each image by selecting one of the numbered figures [11]. A number of works [23, 32 and others] carried out their quantitative assessment of the emotional impact of images from the IAPS database using the method SAM. In these papers emphasize the highly significant correlation of the obtained numerical estimates of valency with the estimates received in [30]. At the same time, the similarities in assessing the intensity of the emotional impact of images from the IAPS database among subjects from different countries are varied.

In our study, the following images were selected from the
IAPS database with valency values characteristic of each type: 10 positive (№ in the IAPS database: 2216, 4250, 4607, $4689,5470,5621,7279,8158,8190,8499$; average valency

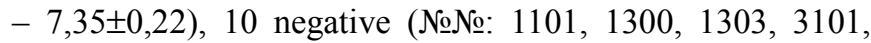
$3185,6350,6370,9140,9908,9941$; average valency $-3,42$ $\pm 0,31)$ and 10 neutral (№№: 2320, 2480, 5740, 7041, 7161, $7179,7205,7700,7705,9360$; average valency $-4,87 \pm 0,14)$. Images were presented to subjects in random order; each image was exposed for 6 seconds; a gray mask was presented between images during 1 second. Presentation of images and synchronization with registration of eye movements was carried out using the EventIDE program (okazolab.com).

Eye movements were recorded using the SMI iView X HiSpeed $1250 \mathrm{~Hz}$ system. The distance between the monitor and the test subject was $50 \mathrm{~cm}$. The images were examined binocularly, but only the movements of one leading eye were recorded. The monitor (NEC MultiSync LCD 1990Sxi), on which images were presented for free viewing, had a frame frequency of $60 \mathrm{~Hz}$ and a resolution of $1280 \times 1024$ pixels. Saccades and gaze fixation were detected using the iView $\mathrm{X}$ package and a computer program implemented in the EventIDE package. The threshold speed of eye movements for the detection of saccades was $40 \%$ s.

Fixations with a duration of less than $100 \mathrm{~ms}$ and more than $1000 \mathrm{~ms}$, as well as saccades with an amplitude of less than $1^{\circ}$ and more than $10^{\circ}$, were excluded from further analysis. Areas of interest were identified by the spatial distribution of all gaze fixations using the modified method of the nearest neighbour [8], the parameters of which were the number of points in the region (at least 3 ) and the distance between the points in one region (not more than $2^{\circ}$ of the field of view). As a result, taking into account the total number of gaze fixations in each test, the regions with the highest fixation density were identified.

Return gaze fixations were determined using the method described in [5]. A fixation was considered to be return one (point " 3 " in Figure 1) if it was located after the returning saccade (grey line in Figure 1) within the foveal neighbourhood (inside a circle with a radius $r=2^{\circ}$ ) of the previous fixation (point " 1 " on Figure 1). Then, the frequency of occurrence (probability) and the coordinates of the return fixations, as well as their density and duration in various areas of the images, were calculated.

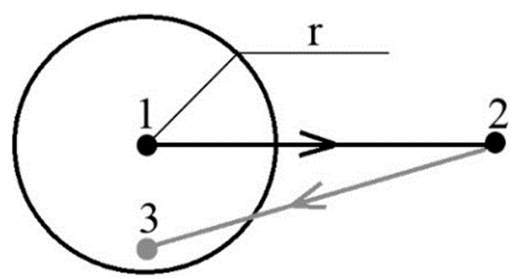

Figure 1. Scheme for defining return fixations (explanations in the text).

Statistical analysis was performed using BeGaze and R: A Language and Environment for Statistical Computing (http://www.R-project.org). The significance of differences between particular samples was determined by the criteria for the sum of Wilcoxon ranks and the t-student test. 


\section{Results}

In each test, the probability (p) of return fixations was calculated as their share among the total number of both regular and return fixation. The averaged data for the three types of images and all subjects are presented in Table 1. It was shown that the average probability of return fixation under free viewing is relatively low for images with different emotional valence. In different tests, individual $\mathrm{p}$ of return fixations varied from 0,04 to 0,42 . The coefficient of variation $\mathrm{p}$ between the subjects was higher at view neutral images (see Table 1).

Figure 2 shows the distribution of the amplitude of the returning saccades (Figure 2, a) and the duration of the return fixations (Figure 2, b) that occur after them. A visual analysis of the distributions indicates their bimodal nature.

An additional analysis using the maximum likelihood estimation algorithm for inhomogeneous distributions (http://www.R-project.org) revealed the presence of two particular lognormal distributions and their statistical parameters (Figure 2, a, b). Highly significant $(\mathrm{p}<0,001$, Student t-tests) differences were found between the average values of the amplitude of the returning saccades $(2,8 \pm 0,1$ and 7,7 $\pm 0,3$ degrees) and the duration of the return fixations $(253 \pm 6 \mathrm{~ms}$ and $600 \pm 20 \mathrm{~ms})$ in the first and second distribution modes. The dependence of the duration of the return fixations on the amplitude of the previous saccade was also analyzed (Figure 2, c). The figure shows a trend to a decrease in the duration of return fixations with an increase in the amplitude of returning saccades.

Table 1. Probability and variability of return fixations during viewing images with different emotional valence.

\begin{tabular}{llll}
\hline \multirow{2}{*}{ Parameters } & Images & \\
\cline { 2 - 3 } & Negative & Neutral \\
\hline Probability of return fixations & $0,090 \pm 0,009$ & $0,079 \pm 0,012$ \\
Coefficient of variation of the probability of return fixations between subjects, $\%$ & 45 & 71 \\
Total number of all fixations, $\mathrm{n}$ & $\mathrm{n}=2439 \quad$ & $\mathrm{n}=2370$ & $53076 \pm 0,009$ \\
\hline
\end{tabular}

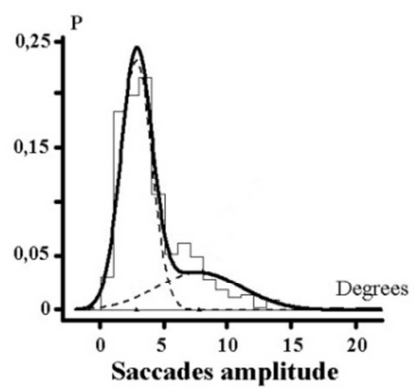

b

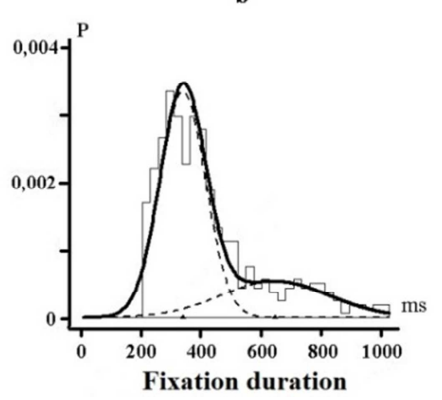

c

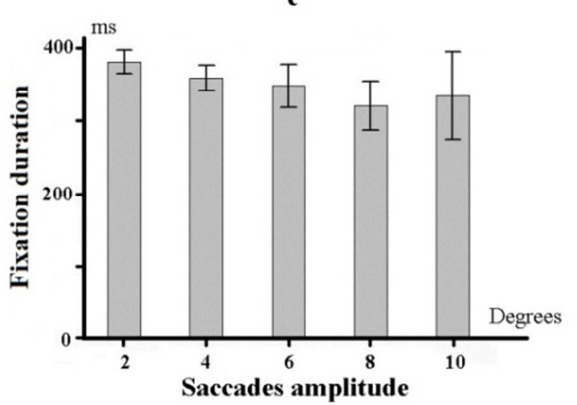

Figure 2. Distributions of amplitudes of returning saccades (a) and durations of return fixations (b). In (a) and (b) the following designations are given: thin solid line - initial distributions; dashed lines - two partial lognormal distributions; wide solid line - the sum of two lognormal distributions; c) the dependence of the duration of return fixations on the amplitude of the returning saccades; vertical notches above each column are standard errors.

Return fixations had a specific spatial distribution and were mainly addressed to areas of interest (or to the most informative areas in the terminology of A. Yarbus [6]). As seen in Figure 3, most of the return fixations $(88 \%$ on average) are located within areas of interest. Moreover, the density of return fixations is significantly higher $(p<0,001$, Student's t-test) within areas of interest than in other areas of images (Table 2). Besides, the data presented in Table 2 indicate a significantly longer duration of return fixations
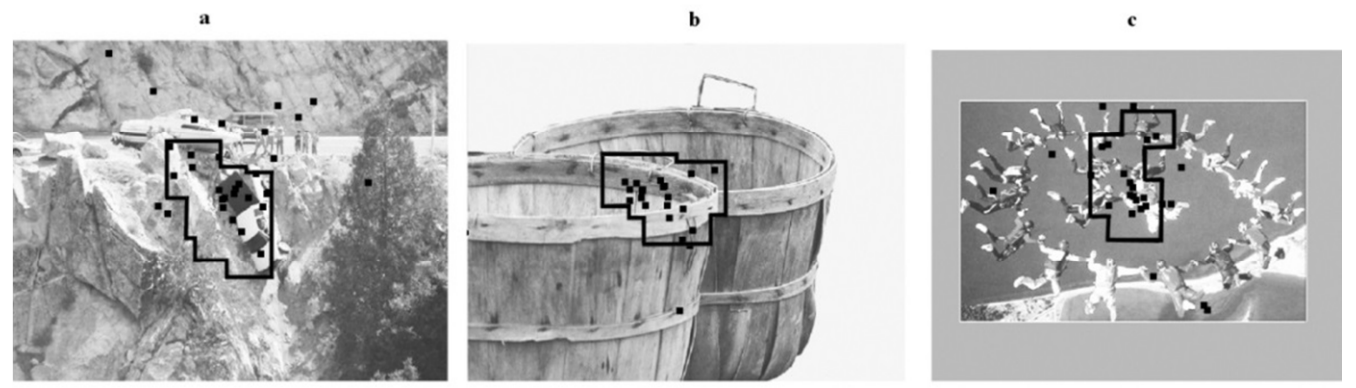

Figure 3. Examples of cumulative maps ( $n=20$ in each case) of the spatial distribution of return fixations (black small squares) while viewing test images; the cumulative ROIs in each image are outlined with black figures: (a) negative (no. 9908 in the IAPS database), (b) neutral (№ 7041) and (c) positive (№ 5621) images. 
Table 2. Average density and duration of return fixations inside and outside areas of interest (AOI).

\begin{tabular}{llll}
\hline \multirow{2}{*}{ Image type } & Density (n/degree $\left.{ }^{2}\right)$ & Duration $(\mathbf{m s e c})$ \\
\cline { 2 - 4 } & Inside AOI & Outside AOI & Inside AOI \\
\hline Negative & $0,019 \pm 0,001$ & $0,006 \pm 0,0001 *$ & $426 \pm 33(\mathrm{n}=189)$ \\
Neutral & $0,022 \pm 0,001$ & $0,006 \pm 0,0001 *$ & $517 \pm 42(\mathrm{n}=158)$ \\
Positive & $0,019 \pm 0,001$ & $0,007 \pm 0,0001 *$ & $475 \pm 46(\mathrm{n}=155)$ \\
\hline
\end{tabular}

The * symbol marks significant differences $(\mathrm{p} \leq 0,001)$ between the mean values of the corresponding parameters within and outside the ROI.

In accordance with the method described in [33], two groups of subjects were distinguished, namely: (1) a group of subjects with the dominance of focal viewing scan-paths (areas of interest were detected in more than $80 \%$ of the tests in 6 subjects); the average share of tests with detected areas of interest is 0,82 ; the average field of the viewing area as a percentage of the total image area is $6,2 \pm 0,6 \%$; (2) a group of subjects with a dominance of scanning viewing scan-paths (areas of interest were detected in less than $40 \%$ of the tests in 6 subjects); the average share of tests with detected areas of interest is 0,23 ; the average field of the viewing area is $25 \pm 2,2 \%$. The subjects with the dominance of focal examination trajectories had a higher probability of return fixations compared with the subjects with the dominance of scanning trajectories $(0,09 \pm 0,003$ and $0,05 \pm 0,006)$. In the group of subjects $(\mathrm{n}=8)$, who occupy an intermediate position between the two polar groups described by the number of tests with detected areas of interest, the average probability of return fixations varied widely - from 0,03 to 0,18 .

\section{Discussion}

In this paper, for the first time, recurrent gaze fixations were discovered during the free viewing of emotionally significant images from the IAPS database. The most important results on the properties of return fixations on the fragments of images just examined are as follows: (a) the distribution of the amplitude of the returning saccades and the duration of the return fixations are bimodal in nature; (b) there is a tendency to increase the duration of return fixations with a decrease in the amplitude of the previous saccade; (c) the location of the return fixations is arranged to areas of interest, inside them return fixations had a significantly greater density and duration than the other parts of the images; (d) subjects with a dominance of focal viewing scanpaths had a higher probability of return fixations than subjects with a dominance of spatially distributed paths.

The average probability of return fixations on recently examined image areas found in our work is close to the known data obtained in the framework of the study of the phenomenon of facilitation of return $[9,11,12]$, despite the use of other methods for identifying return saccades. So, in [12], the probability of return fixations in various tests varied at the level of 0,08 , similar data are given in [11] - from 0,02 to 0,12 in tests for searching and free viewing of complex images. As described in the Introduction, in similar studies using images of a different type, the properties of return saccades and the duration of gaze fixations arising before return saccades [10-12] corresponding to point " 2 " in Figure
1. Based on the results of our previous studies [31] on the peculiarities of fixation duration in their sequence (points " 1 ", " 2 " and " 3 " in Figure 1) in the vicinity of returning saccades during free viewing of reproductions of paintings used in work A Yarbus [6], the fixations occurring after the return saccades are considered as return ones. It was found that the durations of all three fixation in their sequence was significantly longer than regular ones. Moreover, the differences are most pronounced for fixations at point " 3 " and least at point " 1 ". This fact suggests that the planning of return saccades has been predetermined by the results of the analysis of visual information at point " 1 ".

In light of the results obtained, let us consider the possibility of assessing the dominant component of visual attention at the current stage of the dynamic process of viewing images. Previously, a bimodal distribution of the duration of regular fixations was found when solving the task of searching for modified fragments of complex images and joint presentation of targets and distractors $[8,34]$. Moreover, in the latter case, the amplitude of the extremum in the region of long-term fixations has been increased with the increasing complexity of the target stimulus. In both cases, the difference between the average values of the duration of fixations in the two particular distributions was about $200 \mathrm{~ms}$. It is interesting to note that the difference in the average values of the duration of fixations between the first and second modes of distribution in the case of return fixations, found in our work, was of the same order (about $350 \mathrm{~ms}$ ). Apparently, the features of the second extremum in the distribution of fixation durations can be considered as a quantitative measure of the contribution of topdown mechanisms in solving various visual tasks. In turn, quantitative estimates of the contribution of lower-level mechanisms can be received based on an analysis of the characteristics of short-term fixations.

Synchronous antiphase dynamics of the duration of fixations and the amplitude of regular saccades was shown in several works [8, 35-37] at various stages of solving visual tasks, which is considered evidence in favour of a common mechanism of regulation of these parameters of eye movements. Our results (see Figure 2, c) on the joint dynamics of the duration of return fixations and the amplitudes of returning saccades confirm this assumption.

The association of return fixations to the location of areas of interest in images by density and duration parameters found in our study confirms the assumption [21] about the possible participation of return fixations in the activation of short-term memory processes for repeated analysis of image fragments.

Probably, the return fixations should be studied in detail 
using special experimental methods and modelling since they can provide an important key for quantifying the dynamics of visual attention and its interaction with short-term memory mechanisms. In particular, the range of variations of the detected phenomena should be investigated depending on various factors, such as the semantic features of the image and the relationship between objects in the image, the presence of motivation and the current functional state of a person. Like our previous studies $[8,35]$, the temporal dynamics of return fixations during image viewing should be studied in detail.

\section{Conclusion}

It was first discovered the return gaze fixations in the task of free viewing of emotionally significant images from the IAPS database. The most important results in our work on the properties of return fixations on the fragments of images just examined are as follows: (a) the distribution of the amplitude of the returning saccades and the duration of the return fixations are bimodal in nature; (b) there is a tendency to increase the duration of return fixations with a decrease in the amplitude of the previous saccade; (c) the location of the return fixations is arranged to areas of interest, inside them return fixations had a significantly greater density and duration than the other parts of the images; (d) Subjects with a dominance of focal viewing scan-paths had a higher probability of return fixations than Subjects with a dominance of spatially distributed scan-paths. The results obtained allow us to suggest that not only the type of the viewing scan path (the focal or scanning) can be individualizing parameter for quantitative estimation of the dominant type of the visual attention of a particular person but also the probability of return fixations and the fixation duration.

\section{Acknowledgements}

This work is supported by the Ministry of Science and Higher Education of the Russian Federation in the framework of Decree No. 218, project N 2019-218-11-8185 «Creating high-tech production of a software package for managing human capital based on neurotechnology for enterprises in the high-tech sector of the Russian Federation».

\section{References}

[1] Borji, A., \& Itti, L. (2014). Defending Yarbus: Eye movements reveal observers' task. Journal of vision, 14 (3), 29-29.

[2] DeAngelus, M., \& Pelz, J. B. (2009). Top-down control of eye movements: Yarbus revisited. Visual Cognition, 17 (6-7), 790-811.

[3] Podladchikova, L. N., Shaposhnikov, D. G., TikidgjiHamburyan, A. V., Koltunova, T. I., Tikidgji-Hamburyan, R. A., Gusakova, V. I., \& Golovan, A. V. (2009). Model-based approach to study of mechanisms of complex image viewing. Optical Memory and Neural Networks, 18 (2), 114-121.

[4] Privitera, C. M., \& Stark, L. W. (2005). Scanpath theory, attention, and image processing algorithms for predicting human eye fixations. In Neurobiology of Attention (pp. 296299). Academic Press.

[5] Samarin, A., Koltunova, T., Osinov, V., Shaposhnikov, D., \& Podladchikova, L. (2015). Scanpaths of complex image viewing: insights from experimental and modeling studies. Perception, 44 (8-9), 1064-1076.

[6] Yarbus, A. L. (2013). Eye Movements and Vision. Springer. https://doi.org/10.1007/978-1-4899-5379-7

[7] Lupiáñez, J., Klein, R. M., \& Bartolomeo, P. (2006). Inhibition of return: Twenty years after. Cognitive neuropsychology, 23 (7), 1003-1014.

[8] Podladchikova, L. N., Shaposhnikov, D. G., Koltunova, T. I., Dyachenko, A. V., \& Gusakova, V. I. (2009). Temporal dynamics of fixation duration, saccade amplitude, and viewing trajectory. Journal of integrative neuroscience, 8 (4), 487-501.

[9] Dodd, M. D., Van der Stigchel, S., \& Hollingworth, A. (2009). Novelty is not always the best policy: Inhibition of return and facilitation of return as a function of visual task. Psychological Science, 20 (3), 333-339.

[10] Hooge, I. T. C., \& Frens, M. A. (2000). Inhibition of saccade return (ISR): Spatio-temporal properties of saccade programming. Vision research, 40 (24), 3415-3426.

[11] Hooge, I. T. C., Over, E. A., van Wezel, R. J., \& Frens, M. A. (2005). Inhibition of return is not a foraging facilitator in saccadic search and free viewing. Vision research, 45 (14), 1901-1908.

[12] Luke, S. G., Schmidt, J., \& Henderson, J. M. (2013). Temporal oculomotor inhibition of return and spatial facilitation of return in a visual encoding task. Frontiers in psychology, 4, 400 .

[13] Martín-Arévalo, E., Chica, A. B., \& Lupiáñez, J. (2016). No single electrophysiological marker for facilitation and inhibition of return: A review. Behavioural brain research, 300, $1-10$.

[14] Mills, M., Dalmaijer, E. S., Van der Stigchel, S., \& Dodd, M. D. (2015). Effects of task and task-switching on temporal inhibition of return, facilitation of return, and saccadic momentum during scene viewing. Journal of Experimental Psychology: Human Perception and Performance, 41 (5), 1300.

[15] Pratt, J., \& Castel, A. D. (2001). Responding to feature or location: A re-examination of inhibition of return and facilitation of return. Vision Research, 41 (28), 3903-3908.

[16] Posner, M. I., Rafal, R. D., Choate, L. S., \& Vaughan, J. (1985). Inhibition of return: Neural basis and function. Cognitive neuropsychology, 2 (3), 211-228.

[17] Fabius, J. H., Schut, M. J., \& Van der Stigchel, S. (2016). Spatial inhibition of return as a function of fixation history, task, and spatial references. Attention, Perception, \& Psychophysics, 78 (6), 1633-1641.

[18] Hunt, A. R., \& Kingstone, A. (2003). Inhibition of return: Dissociating attentional and oculomotor components. Journal of Experimental Psychology: Human Perception and Performance, 29 (5), 1068.

[19] Wang, Z., Satel, J., \& Klein, R. M. (2012). Sensory and motor mechanisms of oculomotor inhibition of return. Experimental Brain Research, 218 (3), 441-453. 
[20] Weger, U. W., Abrams, R. A., Law, M. B., \& Pratt, J. (2008). Attending to objects: Endogenous cues can produce inhibition of return. Visual Cognition, 16 (5), 659-674.

[21] Bays, P. M., \& Husain, M. (2012). Active inhibition and memory promote exploration and search of natural scenes. Journal of vision, 12 (8), 8-8.

[22] Wang, Z., \& Theeuwes, J. (2012). Dissociable spatial and temporal effects of inhibition of return. PLoS One, 7 (8), e44290.

[23] Barke, A., Stahl, J., \& Kröner-Herwig, B. (2012). Identifying a subset of fear-evoking pictures from the IAPS on the basis of dimensional and categorical ratings for a German sample. Journal of behavior therapy and experimental psychiatry, 43 (1), 565-572.

[24] Bradley, M. M., Houbova, P., Miccoli, L., Costa, V. D., \& Lang, P. J. (2011). Scan patterns when viewing natural scenes: Emotion, complexity, and repetition. Psychophysiology, 48 (11), 1544-1553.

[25] Christianson, S. Å., Loftus, E. F., Hoffman, H., \& Loftus, G. R. (1991). Eye fixations and memory for emotional events. Journal of Experimental Psychology: Learning, memory, and cognition, 17 (4), 693.

[26] Ni, J., Jiang, H., Jin, Y., Chen, N., Wang, J., Wang, Z.,... \& Hu, X. (2011). Dissociable modulation of overt visual attention in valence and arousal revealed by topology of scan path. PLoS One, 6 (4), e18262.

[27] Niu, Y., Todd, R., \& Anderson, A. K. (2012). Affective salience can reverse the effects of stimulus-driven salience on eye movements in complex scenes. Frontiers in psychology, 3, 336.

[28] Pilarczyk, J., \& Kuniecki, M. (2014). Emotional content of an image attracts attention more than visually salient features in various signal-to-noise ratio conditions. Journal of vision, 14 (12), 4-4.

[29] Sabatinelli, D., Lang, P. J., Keil, A., \& Bradley, M. M. (2007).
Emotional perception: correlation of functional MRI and event-related potentials. Cerebral cortex, 17 (5), 1085-1091.

[30] Lang, P., \& Bradley, M. M. (2007). The International Affective Picture System (IAPS) in the study of emotion and attention. Handbook of emotion elicitation and assessment, 29, 70-73.

[31] Lang, P. J., Bradley, M. M., \& Cuthbert, B. N. (2008). International affective picture system (IAPS): affective ratings of pictures and instruction manual. University of Florida, Gainesville. Tech Rep A-8.

[32] Soares, A. P., Pinheiro, A. P., Costa, A., Frade, C. S., Comesaña, M., \& Pureza, R. (2015). Adaptation of the international affective picture system (IAPS) for European Portuguese. Behavior Research Methods, 47 (4), 1159-1177.

[33] Podladchikova, L. N., Koltunova, T. I., Shaposhnikov, D. G., \& Lomakina, O. V. (2017). Individual features of viewing emotionally significant images. Neuroscience and Behavioral Physiology, 47 (8), 941-947.

[34] Koltunova, T. I., Podladchikova, L. N., Shaposhnikov, D. G., Vladimirskii, B. M., Syrkin, L. D., Kryuchkov, B. I., \& Usov, V. M. (2017). Dynamics of the duration of gaze fixation and event-related potentials on presentation of fading-in images and distractors. Neuroscience and Behavioral Physiology, 47 (3), 321-327.

[35] Koltunova, T. I., \& Podladchikova, L. N. (2013). Distractor effect at initial stages of recognition depends on visual image properties. Journal of integrative neuroscience, 12 (01), 91101.

[36] Pannasch, S., Schulz, J., \& Velichkovsky, B. M. (2011). On the control of visual fixation durations in free viewing of complex images. Attention, Perception, \& Psychophysics, 73 (4), 1120-1132.

[37] Unema, P. J., Pannasch, S., Joos, M., \& Velichkovsky, B. M. (2005). Time course of information processing during scene perception: The relationship between saccade amplitude and fixation duration. Visual cognition, 12 (3), 473-494. 